\title{
Chapter 6. Hypertension associated with organ damage
}

\author{
Hypertension Research (2014) 37, 301-314; doi:10.1038/hr.2014.9
}

\section{POINT 6A}

1. In patients undergoing thrombolytic therapy in the hyperacute phase of cerebral infarction, blood pressure should be controlled at $<180 / 105 \mathrm{~mm} \mathrm{Hg}$ within $24 \mathrm{~h}$ after treatment (Recommendation grade: C1, Evidence level: VI).

2. In the hyperacute (within $24 \mathrm{~h}$ after onset) and acute (within 2 weeks after onset) phases of cerebral infarction, for which thrombolytic therapy is not indicated, the target of blood pressure control should be $85-90 \%$ of the pretreatment value when systolic and diastolic blood pressures exceed 220 and $120 \mathrm{~mm} \mathrm{Hg}$, respectively (Recommendation grade: C1, Evidence level: III).

3. In the chronic phase of cerebral infarction (1 month or more after onset), the target level of blood pressure control should be $<140 / 90 \mathrm{~mm} \mathrm{Hg}$ (Recommendation grade: C1, Evidence level: III). In patients with marked stenosis of the bilateral carotid arteries or occlusion of a main trunk of the cerebral arteries, caution against an excessive decrease in blood pressure is particularly necessary (Recommendation grade: C1, Evidence level: V). In patients with lacunar infarction or those taking antithrombotic drugs, a lower level, $<130 /$ $80 \mathrm{~mm} \mathrm{Hg}$, should be targeted if possible (Recommendation grade: $\mathrm{C} 1$, Evidence level: III (lacunar infarction), Evidence level: IVb (antithrombotic drugs)).

4. Cerebral hemorrhage: In the hyperacute (within $24 \mathrm{~h}$ after onset), acute and subacute phases, antihypertensive therapy should be indicated for patients with a systolic blood pressure of $>180 \mathrm{~mm} \mathrm{Hg}$ or a mean blood pressure of $>130 \mathrm{~mm} \mathrm{Hg}$ (Recommendation grade: C1, Evidence level: III). The target of blood pressure control should be $80 \%$ of the pretreatment value (Recommendation grade: $\mathrm{C} 1$, Evidence level: VI). In the chronic phase, the target of blood pressure control should be $<140 / 90 \mathrm{~mm} \mathrm{Hg}$. If possible, a lower level, $<130 / 80 \mathrm{~mm} \mathrm{Hg}$, should be targeted (Recommendation grade: $\mathrm{C} 1$, Evidence level: VI).

5. Subarachnoid hemorrhage: In patients with subarachnoid hemorrhage related to ruptured cerebral aneurysms from onset until the treatment of cerebral aneurysms, antihypertensive therapy should be performed, targeting $80 \%$ of the pretreatment value, when the systolic blood pressure exceeds $160 \mathrm{~mm} \mathrm{Hg}$ (Recommendation grade: C1, Evidence level: $\mathrm{VI})$. In the chronic phase, the target of blood pressure control should be $<140 / 90 \mathrm{~mm} \mathrm{Hg}$. If possible, a lower level, $<130 / 80 \mathrm{~mm} \mathrm{Hg}$, should be targeted (Recommendation grade: $\mathrm{C} 1$, Evidence level: $\mathrm{VI})$.
6. In the hyperacute phase of cerebrovascular disease, the i.v. administration of a very low dose of nicardipine, diltiazem, nitroglycerin or nitroprusside is recommended (Recommendation grade: C1, Evidence level: VI). In the acute phase, it should be switched to oral antihypertensive drugs if possible. The sublingual administration of nifedipine should be avoided, because it may induce a rapid decrease in blood pressure (Recommendation grade: D, Consensus, Evidence level: VI).

7. Oral antihypertensive drugs such as Ca channel blockers, angiotensin-converting enzyme inhibitors, angiotensin II receptor blockers and diuretics are recommended for patients with cerebrovascular disease (Recommendation grade: $\mathbf{C} 1$, Evidence level: VI).

\section{CEREBROVASCULAR DISEASE}

In Japan, cerebrovascular disease accounts for a high percentage of patients with hypertensive organ damage, and the number of patients with cerebrovascular disease, particularly those with cerebral infarction, is increasing with the aging of the population. Many patients with cerebrovascular disease develop hypertension in the acute phase, and blood pressure control in the acute phase is an initial problem. In particular, how antihypertensive therapy should be conducted on reperfusion therapy (thrombolytic therapy or intravascular treatment) for cerebral infarction in the hyperacute phase has also become an important clinical issue. Furthermore, hypertension is the most important risk factor involved in the recurrence of cerebrovascular disease, and blood pressure management for the prevention of recurrence is necessary. In hypertensive patients with cerebrovascular disease, subjects to be treated with antihypertensive drugs and the target level of blood pressure control are determined on the basis of the clinical disease type, interval after onset, severity, age, and use of antithrombotic drugs. In addition, as a high percentage of elderly hypertensive patients are known to have asymptomatic cerebrovascular disease, blood pressure management in hypertensive patients with silent cerebrovascular disease is also important. Treatment for hypertension with cerebrovascular disease is summarized in Table 6-1.

\section{1) Hyperacute/acute phases}

In the hyperacute phase, within $24 \mathrm{~h}$, and in the acute phase, within 1-2 weeks after the onset of cerebrovascular disease, a high blood pressure is observed regardless of the disease type, cerebral infarction, cerebral hemorrhage or subarachnoid hemorrhage. This increase in blood pressure associated with onset is considered to be a biological protective reaction to stress, urinary retention, headache, brain tissue ischemia and an increase in intracranial pressure due to edema and 
Table 6-1 Treatment for hypertension complicated by cerebrovascular diseases

\begin{tabular}{|c|c|c|c|c|c|}
\hline & & & Conditions to treat & Target BP level & Antihypertensive drugs \\
\hline \multirow[t]{6}{*}{$\begin{array}{l}\text { Hyperacute phase } \\
\text { (within } 24 \mathrm{~h} \text { after onset) }\end{array}$} & Cerebral infarction & $\begin{array}{l}\text { Within } 4.5 \mathrm{~h} \\
\text { after onset }\end{array}$ & $\begin{array}{l}\text { Patients awaiting } \\
\text { thrombolytic therapy }{ }^{a}\end{array}$ & $\begin{array}{l}\text { During thrombolytic therapy and } \\
24 \mathrm{~h} \text { after thrombolytic therapy }\end{array}$ & $\begin{array}{l}\text { i.v. instillation of nicardipine, } \\
\text { diltiazem, nitroglycerin or } \\
\text { nitroprusside }\end{array}$ \\
\hline & & & $\begin{array}{l}\mathrm{SBP}>185 \mathrm{~mm} \mathrm{Hg} \text { or } \\
\mathrm{DBP}>110 \mathrm{~mm} \mathrm{Hg}\end{array}$ & $<180 / 105$ mm Hg & \\
\hline & & $\begin{array}{l}\text { Within } 24 \mathrm{~h} \\
\text { after onset }\end{array}$ & $\begin{array}{l}\text { Patients in whom } \\
\text { thrombolytic therapy is } \\
\text { not performed } \\
\text { SBP }>220 \mathrm{~mm} \mathrm{Hg} \text { or } \\
\text { DBP }>120 \mathrm{~mm} \mathrm{Hg}\end{array}$ & $\begin{array}{l}85-90 \% \text { of the pretreatment } \\
\text { value }\end{array}$ & \\
\hline & Cerebral hemorrhage & & $\begin{array}{l}\mathrm{SBP}>180 \mathrm{~mm} \mathrm{Hg} \text { or } \\
\mathrm{MBP}>130 \mathrm{~mm} \mathrm{Hg}\end{array}$ & $80 \%$ of the pretreatment value $\mathrm{e}^{\mathrm{b}}$ & \\
\hline & & & SBP $150-180$ mm Hg & SBP: 140 mm Hg & \\
\hline & $\begin{array}{l}\text { Subarachnoid hemor- } \\
\text { rhage (from the onset of } \\
\text { ruptured cerebral } \\
\text { aneurysm until the } \\
\text { treatment of cerebral } \\
\text { aneurysm) }\end{array}$ & & $\mathrm{SBP}>160 \mathrm{~mm} \mathrm{Hg}$ & $80 \%$ of the pretreatment value ${ }^{c}$ & \\
\hline \multirow[t]{3}{*}{$\begin{array}{l}\text { Acute phase (within } 2 \\
\text { weeks after onset) }\end{array}$} & Cerebral infarction & & $\begin{array}{l}\mathrm{SBP}>220 \mathrm{~mm} \mathrm{Hg} \text { or } \\
\mathrm{DBP}>120 \mathrm{~mm} \mathrm{Hg}\end{array}$ & $\begin{array}{l}85-90 \% \text { of the pretreatment } \\
\text { value }\end{array}$ & $\begin{array}{l}\text { i.v. instillation of nicardipine, } \\
\text { diltiazem, nitroglycerin, or } \\
\text { nitroprusside, or oral drugs } \\
\text { (Ca channel blockers, ACE } \\
\text { inhibitors, ARBs or diuretics) }\end{array}$ \\
\hline & Cerebral hemorrhage & & $\begin{array}{l}\mathrm{SBP}>180 \mathrm{~mm} \mathrm{Hg} \text { or } \\
\mathrm{MBP}>130 \mathrm{~mm} \mathrm{Hg}\end{array}$ & $80 \%$ of the pretreatment value $\mathrm{e}^{\mathrm{b}}$ & \\
\hline & & & SBP $150-180$ mm Hg & SBP: 140 mm Hg & \\
\hline \multirow[t]{4}{*}{$\begin{array}{l}\text { Subacute phase (3-4 } \\
\text { weeks after onset) }\end{array}$} & Cerebral infarction & & $\begin{array}{l}\mathrm{SBP}>220 \mathrm{~mm} \mathrm{Hg} \text { or } \\
\mathrm{DBP}>120 \mathrm{~mm} \mathrm{Hg}\end{array}$ & $\begin{array}{l}85-90 \% \text { of the pretreatment } \\
\text { value }\end{array}$ & $\begin{array}{l}\text { Oral drugs (Ca channel blockers, } \\
\text { ACE inhibitors, ARBs or } \\
\text { diuretics) }\end{array}$ \\
\hline & & & $\begin{array}{l}\text { Patients with an SBP of } \\
180-220 \mathrm{~mm} \mathrm{Hg} \text { in } \\
\text { whom there is no } 50 \% \\
\text { or greater stenosis of } \\
\text { the carotid artery or a } \\
\text { main trunk of the cere- } \\
\text { bral arteries }\end{array}$ & $\begin{array}{l}85-90 \% \text { of the pretreatment } \\
\text { value }\end{array}$ & \\
\hline & Cerebral hemorrhage & & $\begin{array}{l}\mathrm{SBP}>180 \mathrm{~mm} \mathrm{Hg} \\
\mathrm{MBP}>130 \mathrm{~mm} \mathrm{Hg}\end{array}$ & $80 \%$ of the pretreatment value & \\
\hline & & & SBP $150-180 \mathrm{~mm} \mathrm{Hg}$ & SBP: 140 mm Hg & \\
\hline \multirow{2}{*}{$\begin{array}{l}\text { Chronic phase ( } 1 \text { month } \\
\text { or more after onset) }\end{array}$} & Cerebral infarction & & $\mathrm{SBP} \geqslant 140 \mathrm{~mm} \mathrm{Hg}$ & $<140 / 90 \mathrm{~mm} \mathrm{Hg}^{\mathrm{d}}$ & \\
\hline & $\begin{array}{l}\text { Cerebral hemorrhage } \\
\text { Subarachnoid hemor- } \\
\text { rhage }\end{array}$ & & $\mathrm{SBP} \geqslant 140 \mathrm{~mm} \mathrm{Hg}$ & $<140 \mathrm{~mm} \mathrm{Hg}$ & \\
\hline
\end{tabular}

Abbreviations: DBP, diastolic blood pressure; MBP, mean arterial blood pressure; SBP, systolic blood pressure.

aln patients in whom endovascular therapy is scheduled, it should be performed in accordance with thrombolytic therapy.

bIn patients in whom increased intracranial pressure is expected due to a severe condition, cerebral perfusion pressure may reduce with blood pressure, deteriorating symptoms or inducing acute renal dysfunction; therefore, antihypertensive treatment should be performed carefully.

In patients in whom increased intracranial pressure is expected due to a severe condition or those with acute cerebral infarction or cerebrovascular spasm, cerebral perfusion pressure may reduce with blood pressure, deteriorating symptoms; therefore, antihypertensive treatment should be performed carefully.

${ }^{\mathrm{d} B l o o d}$ pressure should be reduced slowly. In patients with marked bilateral carotid artery stenosis or those with occlusion of a main trunk of the cerebral arteries, an excessive decrease in blood pressure should be particularly avoided. In patients with lacunar infarction or those concomitantly taking antithrombotic drugs, a lower level, $<130 / 80 \mathrm{~mm} \mathrm{Hg}$, should be targeted.

eIf possible, the target blood pressure should be $<130 / 80 \mathrm{~mm} \mathrm{Hg}$.

hematoma. In many patients, blood pressure gradually decreases through resting, urination by bladder catheterization, pain control and treatment of brain edema without the administration of antihypertensive drugs. It begins to decrease within $24 \mathrm{~h}$ after onset in most patients with cerebral infarction and within a few days in those with cerebral hemorrhage. ${ }^{536,537}$
The range of autoregulation of cerebral blood flow is shifted to the right due to hypertension. ${ }^{538}$ Autoregulation itself disappears in the acute phase of cerebrovascular disease, and cerebral blood flow decreases even with a slight reduction in blood pressure. Thus, lowering blood pressure further reduces local cerebral blood flow in the lesion and in the surrounding penumbra region (area of reversible 
damage in which functional recovery with restoration of blood pressure is expected), possibly causing enlargement of the lesion (infarction). ${ }^{539}$ As the ischemic area is in a state of vasoparalysis, vasodilator drugs only dilate blood vessels of the intact areas, with a decrease in blood flow in the lesion, which is called intracranial steal. For these reasons, aggressive antihypertensive treatment is not performed, in principle, in the acute phase of cerebrovascular disease. ${ }^{540}$

\section{(1) Target level of blood pressure control}

Cerebral infarction. In the Acute Candesartan Cilexetil Therapy in Stroke Survivors (ACCESS) Study, ${ }^{541}$ patients with cerebral infarction in whom systolic blood pressure (mean value of at least two sessions of blood pressure measurement) was $\geqslant 200 \mathrm{~mm} \mathrm{Hg}$ or diastolic pressure was $\geqslant 110 \mathrm{~mm} \mathrm{Hg} 6$ to $24 \mathrm{~h}$ after admission, or in whom systolic blood pressure was $\geqslant 180 \mathrm{mmHg}$ or diastolic pressure was $\geqslant 105 \mathrm{~mm} \mathrm{Hg} 24$ to $36 \mathrm{~h}$ after admission, were treated with an ARB, candesartan, for 1 week. Although there was no significant difference in the prognosis of stroke, which was a primary end point, the mortality rate after 1 year and occurrence of cardiovascular events, which were secondary end points, significantly reduced. ARBs were expected to have an organ-protecting effect. However, in the Scandinavian Candesartan Acute Stroke Trial (SCAST), ${ }^{542}$ cerebrovascular disease patients (primarily, cerebral infarction, ischemia: 85\%; hemorrhage: $15 \%$ ) with a systolic blood pressure of $>140 \mathrm{~mm} \mathrm{Hg}$ within $30 \mathrm{~h}$ after onset were randomly divided into candesartan-treated and non-candesartan-treated groups for 7-day administration, and the occurrence of complicated cardiovascular events over 6 months was used as a major evaluation item. The blood pressures on day 7 in the candesartan-treated and non-candesartan-treated groups were 147/82 and $152 / 84 \mathrm{~mm} \mathrm{Hg}$, respectively; in the former, the value was significantly lower. There was no significant difference in the complex end point after 6 months between the two groups. The SCAST Study differs from the ACCESS in the following points: subjects with a relatively lower blood pressure were enrolled, and blood pressure was significantly lower in the treatment group. This suggests that antihypertensive treatment should be carefully administered early after the onset of cerebral infarction.

The JSH2014 Guidelines recommend antihypertensive treatment by i.v. administration if systolic blood pressure is $>185 \mathrm{~mm} \mathrm{Hg}$ or diastolic blood pressure is $>110 \mathrm{~mm} \mathrm{Hg}$ in patients in whom thrombolytic therapy by the i.v. injection of tissue plasminogen activator (t-PA) in the hyperacute phase of cerebral infarction, within $4.5 \mathrm{~h}$ after onset, is scheduled according to the Guidelines of the American Stroke Association. ${ }^{536}$ Systolic and diastolic blood pressures should be controlled at $<180$ and $<105 \mathrm{~mm} \mathrm{Hg}$, respectively, by 24 -h strict blood pressure management involving blood pressure monitoring during and after treatment. ${ }^{536}$ Patients in whom endovascular treatment (such as mechanical thrombectomy) is scheduled should also be treated in accordance with thrombolytic therapy.

Even when thrombolytic therapy is not performed, hypertensive encephalopathy, cardiac complications or renal insufficiency may occur in cerebral infarction patients with a systolic blood pressure of $>220 \mathrm{~mm} \mathrm{Hg}$ and a diastolic blood pressure of $>120 \mathrm{~mm} \mathrm{Hg}$; therefore, the target level of blood pressure control should be $85-90 \%$ of the pretreatment value. ${ }^{536}$

Cerebral hemorrhage. There is no sufficient evidence regarding blood pressure management in patients with cerebral hemorrhage. In the Intensive Blood Pressure Reduction in Acute Cerebral Hemorrhage Trial (INTERACT) (pilot study) involving patients with cerebral hemorrhage in the hyperacute phase (within $6 \mathrm{~h}$ after onset), ${ }^{543}$ the results were compared between groups with a target systolic blood pressure of $<140 \mathrm{~mm} \mathrm{Hg}$ and $<180 \mathrm{~mm} \mathrm{Hg}$, and enlargement of hematoma was reduced in the former. However, there was no marked difference in the prognosis. In the INTERACT2 involving 2839 patients with cerebral hemorrhage within $6 \mathrm{~h}$, in which the results were compared between groups with a target systolic blood pressure of $<140 \mathrm{~mm} \mathrm{Hg}$ and $<180 \mathrm{~mm} \mathrm{Hg}$, the mortality rate or incidence of poor outcome (modified Ranking Scale: 3-6) 90 days after onset, as a major evaluation item, was slightly higher in the group with a target systolic blood pressure of $<180 \mathrm{mmHg}$. A shift analysis of the modified Ranking Scale 90 days after onset, as an accessory evaluation item, showed that the functional prognosis was significantly more favorable in the group with a target systolic blood pressure of $<140 \mathrm{~mm} \mathrm{Hg}{ }^{544}$ According to a survey on current blood pressure management for acute-phase cerebral hemorrhage in Japan, ${ }^{545}$ systolic blood pressure control at $\leqslant 160 \mathrm{~mm} \mathrm{Hg}$ by i.v. injection of nicardipine was routinely performed. The results of the SAMURAI-ICH Study, which confirmed the validity of a majority opinion presented in this survey through a multi-center, cooperative, prospective study design, indicated the safety of systolic blood pressure control at $120-160 \mathrm{~mm} \mathrm{Hg}$ by the i.v. injection of nicardipine in Japanese patients. ${ }^{546}$ Its subanalysis showed that blood pressure control at a lower level in the target range of $120-160 \mathrm{~mm} \mathrm{Hg}$ led to a more favorable outcome. ${ }^{547}$

The JSH2014 Guidelines recommend that antihypertensive treatment should be administered to patients with a systolic blood pressure of $>180 \mathrm{~mm} \mathrm{Hg}$ or a mean blood pressure of $>130 \mathrm{~mm} \mathrm{Hg}$ in the hyperacute (within $24 \mathrm{~h}$ after onset) and acute phases of cerebral hemorrhage according to the Guidelines of the American Stroke Association. ${ }^{537}$ The target of blood pressure control should be $80 \%$ of the pretreatment value. In patients with a systolic blood pressure of $150-180 \mathrm{~mm} \mathrm{Hg}$, the target blood pressure should be $\sim 140 \mathrm{~mm} \mathrm{Hg}$. However, the effectiveness of decreasing systolic blood pressure to $140 \mathrm{~mm} \mathrm{Hg}$ or below should be further investigated. In patients in whom an increase in intracranial pressure is expected due to a severe condition, it must be considered that cerebral perfusion pressure may decrease with blood pressure, deteriorating symptoms or causing acute renal insufficiency.

Subarachnoid hemorrhage. In patients with subarachnoid hemorrhage, it is important to prevent additional hemorrhage. Sufficient control of blood pressure, sedation and pain control are desirable. In the Guidelines of the American Stroke Association, ${ }^{548}$ it is described that antihypertensive treatment should be performed when systolic blood pressure is $>160 \mathrm{~mm} \mathrm{Hg}$ in patients with subarachnoid hemorrhage related to ruptured cerebral aneurysms from onset until the treatment of cerebral aneurysms, and that the target level of blood pressure control should be $<160 \mathrm{~mm} \mathrm{Hg}$. The JSH2014 Guidelines recommend that the target of blood pressure control should be $80 \%$ of the pretreatment value when systolic blood pressure exceeds $160 \mathrm{~mm} \mathrm{Hg}$ in patients with subarachnoid hemorrhage related to ruptured cerebral aneurysms from onset until the treatment of cerebral aneurysms. However, it must be considered that cerebral perfusion pressure may decrease with blood pressure, deteriorating symptoms, in patients in whom an increase in intracranial pressure is expected due to a severe condition and in those with acute-phase cerebral infarction or cerebrovascular spasm.

\section{(2) Antihypertensive drugs to be recommended}

Drugs that act quickly and allow dose adjustment are desirable regardless of the clinical disease type of cerebrovascular disease. $\mathrm{Ca}$ 
channel blockers, such as nicardipine and diltiazem or nitroglycerine and nitroprusside, which have long been used, are administered by low-dose i.v. instillation. However, it must be considered that these drugs may increase the intracranial pressure. Furthermore, the sublingual administration of nifedipine capsules should be avoided, because it may induce a rapid decrease in blood pressure. Antihypertensive treatment by injection should be substituted for oral treatment as early as possible. As oral antihypertensive drugs, Ca channel blockers, angiotensin II receptor blockers (ARBs), angiotensin-converting enzyme (ACE) inhibitors and diuretics are recommended.

Rehabilitation from an early stage is necessary for improving activities of daily living in stroke patients, but attention must be paid to changes in blood pressure while conducting rehabilitation at the bedside.

\section{2) Subacute phase}

In the Prevention Regimen for Effectively Avoiding Second Strokes (PRoFESS) Study, ${ }^{549}$ the recurrence rate of stroke, as a primary end point, was followed up for 2.5 years (average) and compared between the telmisartan (ARB, $80 \mathrm{mg}$ per day) and placebo groups in 20332 patients, aged over 55 years, early (median: 15 days) after the onset of ischemic cerebrovascular disease. In the telmisartan group, the mean blood pressure was $3.8 / 2.0 \mathrm{~mm} \mathrm{Hg}$ lower than in the placebo group, but there was no difference in the recurrence rate of stroke. The post hoc analysis ${ }^{550}$ of the PRoFESS showed that, when the mean systolic blood pressure was controlled at $<120 \mathrm{mmHg}$ in patients with noncardiogenic cerebral infarction, the incidence of stroke events was higher than in those in whom it was controlled at $130-139 \mathrm{~mm} \mathrm{Hg}$.

The JSH2014 Guidelines recommend that blood pressure control in the subacute phase of cerebral infarction/hemorrhage, 3 or 4 weeks after onset, should be basically conducted in accordance with that in the acute phase. However, in some patients, blood pressure can be decreased to the level targeted in the chronic phase while evaluating the patient's symptoms. Among patients of cerebral infarction with stable symptoms and systolic blood pressure of $180-220 \mathrm{mmHg}$ in whom stenosis of the carotid artery or of a main trunk of the cerebral arteries is less than $50 \%$ and there may be no possibility of hemodynamic cerebral ischemia related to a decrease of blood pressure, attention must be paid of signs of cerebral circulatory insufficiency during antihypertensive therapy. Target of blood pressure should be $85-90 \%$ of the pretreatment value.

\section{3) Chronic phase}

Patients with a history of cerebrovascular disease are known to frequently develop new cerebrovascular disease, and the control of hypertension, which is its greatest risk factor, is extremely important for the treatment of patients in the chronic phase of cerebrovascular disease. According to the results of a retrospective study in Japan, the relationship between blood pressure after cerebrovascular disease and recurrence rate varies markedly among disease types, and the report of a J-shaped relationship between the recurrence of cerebral infarction and diastolic pressure, which is not observed in patients with cerebral hemorrhage, has attracted attention. ${ }^{551}$

Since 1990, large-scale studies on the relationship between the prevention of recurrent cerebrovascular disease and blood pressure have been carried out, ${ }^{256,552-557}$ with systematic reviews. ${ }^{558,559}$ Antihypertensive drug therapy significantly reduces the recurrence rate of all types of cerebrovascular disease, the recurrence rate of nonfatal cerebral infarction, and incidences of myocardial infarction and all vascular events.

\section{(1) Target of blood pressure control}

Cerebral infarction. In the Perindopril Protection against Recurrent Stroke Study (PROGRESS), ${ }^{256}$ perindopril (4 mg per day) or a diuretic, indapamide ( $2 \mathrm{mg}$ per day), was additionally administered to 6105 patients with chronic-phase cerebrovascular disease, primarily including cerebral infarction (cerebral infarction: 71\%; transient cerebral ischemic attacks: 22\%; and cerebral hemorrhage: $11 \%$ ), with a mean age of 64 years, in addition to conventional treatment. The blood pressure decreased from $147 / 86$ to about $138 / 82 \mathrm{~mm} \mathrm{Hg}$, and the recurrence rate of stroke was reduced by $28 \%$. Its subanalysis $^{232}$ indicated that the incidences of cerebral hemorrhage and cerebral infarction were lower in patients in whom blood pressure was controlled at a lower level (a systolic blood pressure of $\sim 120 \mathrm{~mm} \mathrm{Hg}$ ).

Rothwell et al. ${ }^{282}$ reported that the risk of cerebrovascular disease significantly increased in a group in which the systolic blood pressure decreased to $140 \mathrm{~mm} \mathrm{Hg}$ among patients with symptomatic, $70 \%$ or greater stenosis of the bilateral carotid arteries (accounting for 2-3\%), whereas there was no increase in this risk even when the systolic blood pressure decreased to $140 \mathrm{~mm} \mathrm{Hg}$ in patients with $70 \%$ or greater unilateral carotid artery stenosis. In the Warfarin-Aspirin Symptomatic Intracranial Disease (WASID) Study, ${ }^{560}$ among patients with symptomatic intracranial artery (internal carotid, middle cerebral, vertebral or basilar artery) stenosis, the blood pressure level was not associated with the risk for ischemic cerebrovascular disease in those with marked ( $70 \%$ or greater) stenosis. In those with moderate $(69 \%$ or lower) stenosis, the risk for ischemic cerebrovascular disease was high when the systolic blood pressure was $160 \mathrm{~mm} \mathrm{Hg}$ or above. Thus, in the presence of obstruction of a main trunk of the cerebral arteries or marked stenosis, management matched to individual patients is necessary. Furthermore, the hemodynamics may differ between vascular stenosis and obstruction. There is little evidence as a reference for the relationship between blood pressure and the risk for ischemic cerebrovascular disease in patients with unilateral obstruction of the internal carotid or basilar artery. Yamauchi et al. ${ }^{561}$ examined the relationship between blood pressure and recurrent stroke during follow-up with respect to the presence or absence of impaired perfusion on positron emission tomography in patients with symptomatic atherosclerotic occlusion of a main trunk of the cerebral arteries. In patients with impaired perfusion, the risk of recurrent stroke was high when the systolic blood pressure was $<130 \mathrm{~mm} \mathrm{Hg}$. In those without impaired perfusion, this risk may be high at a high blood pressure level. They concluded that it is important to evaluate the presence or absence of impaired perfusion for blood pressure management.

In the Secondary Prevention of Small Subcortical Strokes (SPS3) Trial, ${ }^{557}$ a randomized comparative study was conducted on 3020 patients with chronic-phase lacunar infarction by dividing them into two groups: a group with a systolic blood pressure of $<130 \mathrm{~mm} \mathrm{Hg}$ (aggressive therapy group) and that with a systolic blood pressure of 130-149 mm Hg (standard therapy group), with a mean follow-up of 3.7 years. The type of antihypertensive drug was not limited. The mean systolic blood pressures after 1 year in the two groups were 127 and $138 \mathrm{~mm} \mathrm{Hg}$, respectively. As a major evaluation item, cerebral infarction/hemorrhage was observed in $152(2.8 \% \mathrm{yr})$ and $125(2.2 \% y r)$ patients in the standard and aggressive therapy groups, respectively; there was no significant difference. Cerebral infarction was present in $131(2.4 \% y r)$ and $112(2.0 \% y r)$ patients, respectively, showing no significant difference. Cerebral hemorrhage was present in $16(0.29 \% y r)$ and $6(0.11 \% y r)$ patients, respectively; its incidence was significantly lower in patients with a lower systolic blood pressure. 
In the Bleeding with Antithrombotic Therapy (BAT) Study, ${ }^{325} 4009$ patients taking antithrombotic drugs for the prevention of recurrent cerebrovascular or heart disease were prospectively enrolled, and hemorrhagic events were investigated over 19 months. Cerebral hemorrhage was observed in $0.3 \%$ of patients taking a single antithrombotic drug, $0.6 \%$ of those receiving combination therapy with an antiplatelet drug, $0.6 \%$ of those taking Warfarin and $1.0 \%$ of those receiving a combination of Warfarin and an antiplatelet drug. The onset of cerebral hemorrhage was particularly noted in patients with a history of cerebrovascular disorder. The results suggest that the incidence of cerebral hemorrhage is lower when the blood pressure immediately before/after onset is lower in patients taking antithrombotic drugs, and that blood pressure should be reduced to $<130 / 81$ mm Hg. ${ }^{323}$

The Guidelines of the American Stroke Association ${ }^{562}$ propose no clear target of blood pressure control or degree of blood pressure reduction and consider that they vary among individual patients. A mean decrease in blood pressure of about $10 / 5 \mathrm{~mm} \mathrm{Hg}$ is effective. The JNC7 emphasizes that a normal blood pressure is defined as $<120$ / $80 \mathrm{~mm} \mathrm{Hg}$.

On the other hand, the ESH/ESC Guidelines revised in $2013^{120}$ recommend a systolic blood pressure of $<140 \mathrm{~mm} \mathrm{Hg}$ as a target of blood pressure control for patients in the chronic phase of cerebrovascular disease. They also propose that a higher target level may be established for elderly patients.

In the JSH2014 Guidelines, the target of blood pressure control for patients with chronic-phase cerebral infarction is established as $<140 / 90 \mathrm{mmHg}$. In particular, an excessive decrease in blood pressure should be avoided in patients with marked stenosis of the bilateral carotid arteries or occlusion of a main trunk of the cerebral arteries. However, the Guidelines recommend that a lower level $(<130 / 80 \mathrm{~mm} \mathrm{Hg})$ should be targeted in patients with lacunar infarction or in those taking antithrombotic drugs (excluding those with $50 \%$ or greater stenosis/occlusion of the carotid artery or a main trunk of the cerebral arteries). Complaints of dizziness, light headedness, tiredness, numbness, weakness, loss of energy or exacerbation of neurological signs or symptoms during treatment may be symptoms of cerebral circulatory insufficiency due to a decrease in blood pressure, and a decrease in the dose or change in the type of antihypertensive drug is necessary. Particular caution is needed in patients with occlusion of a main trunk of the cerebral arteries (especially in the vertebral-basilar artery system), because dysautoregulation of the cerebral circulation may persist for 3 months or more. 563

Cerebral hemorrhage. With regard to recurrent cerebral hemorrhage, a study reported that a diastolic blood pressure of $75-90 \mathrm{~mm} \mathrm{Hg}$ was favorable, ${ }^{551}$ and another study indicated that the recurrence rate was low when diastolic blood pressure was controlled at $<90 \mathrm{~mm} \mathrm{Hg} .{ }^{564}$ However, the evidence level is low. In the Guidelines of the American Stroke Association, ${ }^{537}$ the final target of blood pressure control in patients with cerebral hemorrhage (chronic phase) is established as $<140 / 90 \mathrm{~mm} \mathrm{Hg}$ (diabetes mellitus and chronic kidney disease (CKD): $<130 / 80 \mathrm{mmHg}$ ). The JSH2014 Guidelines recommend that the final target of blood pressure control in patients with cerebral hemorrhage should be $<140 / 90 \mathrm{~mm} \mathrm{Hg}$, and that a blood pressure of $<130 / 80 \mathrm{~mm} \mathrm{Hg}$ should be targeted if possible (excluding patients with $50 \%$ or greater stenosis of the carotid artery or a main trunk of the cerebral arteries and those with obstruction).

Subarachnoid hemorrhage. There is no evidence on the target of blood pressure control in the chronic phase of subarachnoid hemor- rhage. The target of blood pressure control was established in accordance with that in patients with cerebral hemorrhage.

\section{(2) Recommended classes of antihypertensive drug}

In PROGRESS, ${ }^{256}$ a combination of an ACE inhibitor and a diuretic was suggested to reduce the recurrence rate of cerebrovascular disease and prevent the occurrence of dementia. In the Morbidity and Mortality After Stroke (MOSES) Study, ${ }^{556}$ primary end points (all deaths and all cardiovascular and cerebrovascular events) and cerebrovascular events (one of the secondary end points) were significantly lower in the ARB (eprosartan) group than in the Ca channel blocker (nitrendipine) group in spite of no difference in blood pressure reduction between two groups.

The Guidelines of the American Stroke Association ${ }^{562}$ recommend a diuretic alone and a diuretic+ACE inhibitor. Proposing that drugs should be selected for each patient depending on background factors (extracranial obstructive vascular diseases, renal disorders, heart disease and diabetes), they also recommend ARBs and ACE inhibitors for patients with diabetes mellitus or atrial fibrillation. In the 2013 ESHESC Guidelines, ${ }^{120}$ all classes of antihypertensive drugs are recommended, because they consider that most of the benefit obtained from drugs can be ascribed to a decrease in blood pressure. Although differences among drugs are considered to be masked if a strict target of blood pressure control is attained, there are also results indicating differences among drugs despite a similar decrease in blood pressure, such as those shown by MOSES. ${ }^{556}$

Considering the above evidence, the JSH2014 Guidelines recommend Ca channel blockers, ARBs, ACE inhibitors and diuretics, which are first-choice drugs, regardless of the clinical disease type of cerebrovascular disease for the treatment of hypertension in the chronic phase. These drugs do not reduce cerebral blood flow on a decrease in blood pressure (unless there is an excessive decrease in blood pressure). Furthermore, antihypertensive drugs should be selected, considering the presence or absence of associated disorders such as diabetes mellitus, metabolic syndrome and CKD.

\section{4) Asymptomatic phase}

The diagnostic criteria (for brain-dock) issued in $1997^{565}$ are used for the diagnosis of asymptomatic cerebrovascular disease. Most of the silent cerebral infarction closely related to hypertension is a small lesion similar to lacunar infarction, a small vessel disease for which hypertension and age are considered to be the greatest risk factors. Its presence and progression are independent risk factors for cerebrovascular disease and impairment of cognitive function. ${ }^{186,187,566}$ Asymptomatic cerebral hemorrhage (microhemorrhage), which is detected mostly by T2-weighted MRI, is attracting attention. ${ }^{567-569}$

In principle, target of blood pressure and useful antihypertensive drugs for hypertensive patients with silent cerebral infarction or cerebral hemorrhage are the same as those for chronic phase of cerebrovascular disease, but the results of a CT substudy of PROGRESS $^{570}$ suggested that more antihypertensive treatment is desirable. Silent cerebral infarction is an index of target organ damage along with white matter lesions, and non-dipper, rise and morning surges observed by 24 -h blood pressure monitoring are its risk factors. ${ }^{137,151,152,571}$ Blood pressure control over $24 \mathrm{~h}$ and early in the morning is important.

In addition, asymptomatic carotid artery stenosis and unruptured cerebral aneurysms are also frequently detected, and they have been shown to be risk factors for the occurrence of cerebrovascular disease. With regard to asymptomatic carotid artery stenosis, the evaluation of indications for surgical treatment before the initiation 
of antihypertensive treatment is important. If the patient has a familial history of subarachnoid hemorrhage or unruptured cerebral aneurysm, aggressive antihypertensive treatment is recommended.

In the asymptomatic phase, patients feel high-level anxiety over the condition of cerebrovascular disease and treatment, and hence sufficient informed consent is extremely important.

\section{HEART DISEASE}

\section{POINT 6B}

Coronary artery disease

1. In patients with coronary artery disease, it is important to sufficiently reduce blood pressure while paying attention to ischemic symptoms/findings of damages of the heart (Table 6-2). The target of blood pressure control should be $<140 / 90 \mathrm{~mm} \mathrm{Hg}$, in principle (Recommendation grade: A, Evidence level: I).

2. Hypertension complicated by angina pectoris due to organic coronary artery stenosis is a good indication for Ca channel blockers and $\beta$-blockers with no endogenous sympathomimetic action (Recommendation grade: A, Evidence level: I).

3. Vasospastic angina pectoris is a good indication for $\mathrm{Ca}$ channel blockers (Recommendation grade: B, Evidence level: II).

4. In patients with old myocardial infarction, $\beta$-blockers, reninangiotensin (RA) system inhibitors (ACE inhibitors, ARBs) and aldosterone antagonists reduce the mortality rate and improve the prognosis (Recommendation grade: A, Evidence level: I).

Heart failure

1. In patients with heart failure, antihypertensive drugs are used not only to reduce blood pressure but also to improve their quality of life and/or prognosis (Recommendation grade: A, Evidence level: I).

2. The combination of RA system inhibitor+ $\beta$-blocker+diuretic is a standard treatment method for heart failure with reduced ejection fraction (HFrEF). It reduces mortality and improves the prognosis. However, RA system inhibitors and $\beta$-blockers should be started at low doses, and their doses should be titrated carefully and gradually, to avoid the exacerbation of heart failure, hypotension, bradycardia ( $\beta$-blockers) and renal dysfunction (Recommendation grade: A, Evidence level: I).

3. Aldosterone antagonists further improve the prognosis of patients with severe HFrEF on standard treatment (Recommendation grade: A, Evidence level: I).

4. If a decrease in blood pressure is insufficient in patients with HFrEF, a long-acting Ca channel blocker should be added (Recommendation grade: A, Evidence level: I).

5. In patients with heart failure with preserved ejection fraction, sufficient antihypertensive treatment is important (Recommendation grade: B, Evidence level: II).

Cardiac hypertrophy

1. The regression of cardiac hypertrophy leads to an improvement in prognosis (Recommendation grade: A, Evidence level: I).
Table 6-2 Treatment for hypertension complicated by heart disease

Angina Organic coronary stenosis ${ }^{\mathrm{a}}$ : $\beta$-blockers, long-acting Ca channel
pectoris blockers

Coronary vasospasm: long-acting Ca channel blockers If a decrease in blood pressure is insufficient, an RA system inhibitor (ACE inhibitor, ARB) is added.

Old myocardial RA system inhibitors or $\beta$-blockers are the first choice. infarction

If a decrease in blood pressure is insufficient, a long-acting $\mathrm{Ca}$ channel blocker or diuretic is added.

Patients with systolic dysfunction: an aldosterone antagonist is added $^{\mathrm{b}}$.

Heart failure Heart failure with reduced ejection fraction

Standard treatment: RA system inhibitor ${ }^{c}+\beta$-blocker ${ }^{c}+$ diuretic Severe heart failure patients: an aldosterone antagonist is added If a decrease in blood pressure is insufficient, a long-acting $\mathrm{Ca}$ channel blocker is added.

Heart failure with preserved ejection fraction

A sustained and sufficient decrease in blood pressure is important.

Cardiac A sustained and sufficient decrease in blood pressure is necessary.

hypertrophy

RA system inhibitors or long-acting Ca channel blockers are the first choice.

Abbreviation: RA, renin-angiotensin.

aCoronary revascularization is performed in patients with significant organic coronary stenosis. ${ }^{\mathrm{b}} \mathrm{Be}$ aware of hyperkalemia.

cAdministration should be started at a low dose, and the dose should be titrated carefully and slowly.

2. Sustained and sufficient decrease in blood pressure by any antihypertensive drug can induce the regression of cardiac hypertrophy. In particular, RA system inhibitors and Ca channel blockers are effective for cardiac hypertrophy (Recommendation grade: A, Evidence level: I).

The heart is one of the important target organs of hypertension. Increases in systolic and diastolic pressure loads induce myocardial remodeling, such as cardiac hypertrophy and myocardial interstitial fibrosis, and coronary endothelial damage. Risk factors such as dyslipidemia, diabetes mellitus and smoking increase the risk of coronary atherosclerosis and myocardial ischemia. The progression of coronary atherosclerosis and myocardial remodeling leads to coronary artery disease, heart failure, arrhythmia and sudden death.

Atrial fibrillation, of which the incidence has recently increased, markedly increases the risk of cardiogenic cerebral embolism, and the incidence of cardiovascular events and mortality rate increase by about 2.5-fold. ${ }^{572,573}$ Hypertension is the most important risk factor for new onset of atrial fibrillation..$^{574,575}$ In particular, left ventricular hypertrophy and left atrial enlargement are independent risk factors for new onset of atrial fibrillation. When antihypertensive treatment leads to the regression of left ventricular hypertrophy, the incidence of atrial fibrillation decreases. ${ }^{576}$ Furthermore, hypertension also increases the risks of stroke and arterial embolism in patients with chronic atrial fibrillation. ${ }^{577,578}$

In hypertensive patients with heart disease, the number of those taking antithrombotic drugs (antiplatelet drugs, anticoagulants) has increased. As these drugs increase the incidence of hemorrhagic complications, especially intracranial hemorrhage, strict blood pressure control is necessary in patients taking antithrombotic drugs (see Section 7 of Chapter 3, OTHER POINTS REQUIRING ATTENTION). 
Therefore, in hypertensive patients with heart disease, sufficient reduction in blood pressure is mandatory to reduce the cardiovascular mortality rate and incidence of cardiovascular events. ${ }^{237,260,466,579}$

\section{1) Coronary artery disease}

Hypertension increases the incidence of coronary artery disease. However, conventional antihypertensive drug therapy with diuretics and $\beta$-blockers does not markedly reduce the incidence of coronary artery disease. ${ }^{580}$ Recent studies have reported that long-acting Ca channel blockers and RA system inhibitors (ACE inhibitors and ARBs) reduce the incidence of coronary artery disease. ${ }^{446,555,581-583}$ Furthermore, clinical trials in Japan have suggested that cardiac events can be prevented in patients with coronary artery disease by sufficiently reducing blood pressure using RA system inhibitors or long-acting Ca channel blockers. ${ }^{466,584,585}$

To prevent the occurrence and progression of coronary artery disease, management of other risk factors in addition to antihypertensive treatment is important. Particularly, the treatment of hypercholesterolemia using 3-hydroxy-3-methyl-glutaryl coenzyme A (HMG$\mathrm{CoA}$ ) reductase inhibitors, antiplatelet therapy with low-dose aspirin and cessation of smoking have been shown to be useful for the primary and, in particular, secondary prevention of coronary events. $273,483,586-588$

Evidence regarding the target level of blood pressure control in hypertensive patients with coronary artery disease is insufficient. There is also concern that, in these patients, decrease in blood pressure below a certain level causes a reduction in the diastolic coronary perfusion pressure and induces myocardial ischemia, resulting in worsening of the prognosis (J-shaped curve phenomenon). However, these reports were based on retrospective analyses ${ }^{281,589-591}$ and have not been validated. The INVEST retrospective analysis, which suggests the J-shaped curve phenomenon, showed that coronary revascularization reduced a low diastolic blood pressure-related increase in risk by $50 \%$ or more. ${ }^{281}$ A subanalysis of a large-scale registration study (CREDO-Kyoto Registry) including patients after coronary revascularization in Japan indicated that severe arteriosclerosis, CKD and systolic dysfunction rather than a low blood pressure level itself were independent risk factors for cardiovascular mortality, although the crude cardiovascular mortality rate increased in patients with a diastolic blood pressure of $<70 \mathrm{~mm} \mathrm{Hg} .{ }^{592}$ On the other hand, in the EUROPA, ACTION, CAMELOT and NORMALISE placebo-controlled studies including patients with coronary artery disease, the risk of coronary artery disease was reduced and coronary plaques were regressed when systolic blood pressure was decreased from $140-149 \mathrm{~mm} \mathrm{Hg}$ to $130-139 \mathrm{~mm} \mathrm{Hg}$ and from $130-139 \mathrm{~mm} \mathrm{Hg}$ to 120-129 mm Hg. ${ }^{446,454,581,583}$ On the basis of the results of the ACTION and JMIC-B trials, the JSH2014 Guidelines recommend that the target level of blood pressure control in patients with coronary artery disease be $<140 / 90 \mathrm{~mm} \mathrm{Hg} .{ }^{446,585,593}$ Although evidence is not sufficient, the risk of cardiovascular events is even higher in patients with multiple risk factors such as a history of myocardial infarction, diabetes mellitus, CKD, dyslipidemia, smoking and family history. Therefore, in such patients, a lower level $(<130 / 80 \mathrm{~mm} \mathrm{Hg})$ is desirable in the absence of significant coronary artery stenosis, symptoms of myocardial ischemia and ischemic electrocardiographic findings.

\section{(1) Angina pectoris}

In hypertensive patients with angina pectoris, Ca channel blockers and $\beta$-blockers are the first choice because of their antianginal actions. Angina pectoris is caused by significant coronary stenosis, coronary vasospasm or both. As coronary vasospasm is prevented by Ca channel blockers, they are the first choice for hypertension complicated by angina at rest and rest-and-effort angina. ${ }^{594-596}$ Both $\beta$-blockers and Ca channel blockers are effective for angina on effort due to organic coronary artery stenosis. ${ }^{597,598}$ In Japan, angina pectoris attributed to coronary vasospasm is frequently observed. As $\beta$-blockers have been suggested to exacerbate coronary vasospasm, a Ca channel blocker or a combination of a Ca channel blocker and a $\beta$-blocker is recommended when the mechanism of angina pectoris is unclear.

As the antianginal actions of $\beta$-blockers are attributable primarily to heart-rate-lowering effects, $\beta$-blockers that do not possess endogenous sympathomimetic actions should be selected. ${ }^{599}$ There is no difference in antianginal effects between $\beta 1$-selective and nonselective drugs. Furthermore, the blood-pressure-lowering effect of $\beta$-blockers is sometimes not enough; ${ }^{600,601}$ therefore, when a decrease in blood pressure is insufficient, combination therapy with a long-acting $\mathrm{Ca}$ channel blocker or an RA system inhibitor is necessary.

Among Ca channel blockers, long-acting dihydropyridine Ca channel blockers and diltiazem sustained-release preparations are recommended, because reflex tachycardia associated with a decrease in blood pressure is observed less frequently and because the time of administration need not be adjusted for the time of occurrence of anginal attacks. With regard to short-acting dihydropyridine Ca channel blockers, a rapid decrease in blood pressure or reflex tachycardia may induce myocardial ischemia in patients with flow-limiting coronary artery stenosis.

In patients with angina pectoris due to significant coronary artery stenosis, coronary revascularization (coronary bypass grafting or percutaneous coronary intervention) is effective for reducing anginal pain and for decreases in cardiac events related to a low diastolic blood pressure. ${ }^{281}$ Therefore, when patients have chest pain-like symptoms it is advisable to ask cardiologists to evaluate coronary artery stenosis/ myocardial ischemia.

\section{(2) Old myocardial infarction}

In large-scale clinical studies in Western countries, $\beta$-blockers with no endogenous sympathomimetic action were found to significantly reduce the recurrences of myocardial infarction and sudden death in patients with old myocardial infarction. ${ }^{602,603}$ In particular, $\beta$-blockers should be used for the secondary prevention of coronary artery disease in patients with old myocardial infarction associated with systolic dysfunction and in those with myocardial infarction/acute coronary syndrome within 3 years after onset. ${ }^{587,588}$ In Japan, $\beta$-blockers are used less frequently, partly because of concern about coronary vasospasm. However, in patients with old myocardial infarction having significant organic coronary artery lesions, $\beta$-blockers are a treatment option. Short-acting $\mathrm{Ca}$ channel blockers may increase cardiac accidents, but long-acting Ca channel blockers do not worsen the prognosis. ${ }^{237}$ In addition, diltiazem reduced the recurrence of myocardial infarction in patients with non-Q-wave infarction without heart failure. ${ }^{604}$ In follow-up studies of a large number of patients conducted in Japan, both $\beta$-blockers and long-acting Ca channel blockers were found to reduce the incidence of cardiac events, ${ }^{585,605-607}$ but short-acting Ca channel blockers tended to exacerbate them ${ }^{608}$ in patients with old myocardial infarction.

In patients with left ventricular systolic dysfunction after myocardial infarction (ejection fraction: $\leqslant 40 \%$ ), RA system inhibitors have been shown to prevent left ventricular remodeling (ventricular dilation, myocardial hypertrophy and interstitial fibrosis) and reduce the incidence of heart failure and sudden death. ${ }^{609,610}$ Ventricular remodeling has an important role in the progression of myocardial damage 
and in the occurrence/exacerbation of heart failure. Therefore, left ventricular dilation and systolic dysfunction after myocardial infarction are good indications for RA system inhibitors. Many large-scale clinical studies have also indicated that ACE inhibitors decrease the incidence of cardiovascular complications after myocardial infarction, while improving the prognosis; their usefulness in the secondary prevention of myocardial infarction has been established. ${ }^{609,611,612}$ The Guideline for the Secondary Prevention of Myocardial Infarction published by the Japanese Circulation Society states that, among RA system inhibitors, ACE inhibitors are preferred as a first-choice drug for the secondary prevention of myocardial infarction and that ARBs may be used only when there is no tolerance to ACE inhibitors. ${ }^{483}$ In patients with a severe systolic dysfunction after myocardial infarction, the prognosis is further improved by the administration of an aldosterone antagonist in addition to an RA system inhibitor, a $\beta$ blocker and a diuretic. ${ }^{499,613}$

\section{2) Heart failure}

Epidemiological studies in Western countries have shown that hypertension is the most frequent underlying cause of heart failure, and similar results have been obtained in a patient registration study in Japan. ${ }^{614}$ Large-scale clinical studies in Western countries have also shown that antihypertensive treatment reduces the incidence of heart failure in hypertensive patients. ${ }^{615}$

Many patients with heart failure have normal or low blood pressure. Therefore, in patients with heart failure, antihypertensive drugs are not necessarily used for reducing blood pressure but, more importantly, for improving their quality of life, preventing hospitalization due to heart failure and improving the prognosis.

\section{(1) Heart failure with reduced ejection fraction}

RA system inhibitors improve the long-term prognosis of chronic heart failure and myocardial infarction and reduce the frequency of hospitalization regardless of the presence or absence of symptoms of heart failure or the degree of left ventricular dysfunction. ${ }^{609-613,616-622}$ In the Guidelines for the Treatment of Chronic Heart Failure published by the Japanese Circulation Society, it is recommended that, among RA system inhibitors, ACE inhibitors should be initially used to treat heart failure with reduced ejection fraction (HFrEF) and that ARBs should be used when there is no tolerance to ACE inhibitors. ${ }^{447}$ Treatment with $\beta$-blockers should be started at a low dose and doses are titrated gradually with caution. $\beta$-blockers improve the prognosis of patients with HFrEF and reduce the frequency of hospitalization regardless of the presence or absence of symptoms. ${ }^{63,623-626}$ Furthermore, diuretics are used for the treatment and prevention of organ congestion. Therefore, combination therapy with an RA system inhibitor, a $\beta$-blocker and a diuretic is a standard treatment method for systolic dysfunction. ${ }^{447,627-629}$ Moreover, the addition of an aldosterone antagonist further improves the prognosis of patients with severe HFrEF on the standard treatment. ${ }^{499,613,616}$

In large-scale clinical studies, the doses of RA system inhibitors and $\beta$-blockers that improved the prognosis of heart failure were higher than those used for the treatment of hypertension in Japan. However, as the RA system is activated in heart failure patients, RA system inhibitors exhibit marked hypotensive effects. Therefore, their administration should be started at a low dose (for example, $1 / 4$ to $1 / 2$ of a tablet regardless of the dosage form), and the dose should be gradually increased by confirming the absence of adverse effects such as hypotension and renal dysfunction. In addition, the use of $\beta$-blockers should be attempted as much as possible after RA system inhibitors regardless of the severity of heart failure, but utmost caution is necessary at the beginning of their use because $\beta$-blockers may exacerbate heart failure. In patients with a reduced systolic function, the administration of $\beta$-blockers should be started at a very low dose (1/8 to $1 / 4$ of the dose for hypertension), and the dose should be increased slowly by confirming the absence of heart failure, bradycardia and hypotension.

In hypertensive patients with HFrEF, the treatment of hypertension is important, because hypertension aggravates heart failure by augmenting the left ventricular afterload. In addition, as hypertension promotes left ventricular remodeling and the progression of myocardial damage, hypertension treatment is important for improving the long-term prognosis. Long-acting dihydropyridine Ca channel blockers have been shown not to worsen the prognosis of heart failure patients. ${ }^{237,630,631}$ Therefore, if a sufficient blood-pressure-lowering effect cannot be obtained with antihypertensive drugs used for the standard treatment of heart failure, a dihydropyridine $\mathrm{Ca}$ channel blocker may be added.

\section{(2) Heart failure with preserved ejection fraction}

Impairment of diastolic, but not systolic, function is the primary cause of heart failure in nearly a half of the patients hospitalized due to heart failure. Hypertensive heart disease is the most frequent underlying disease for heart failure with preserved ejection fraction (HFpEF), particularly in elderly and female patients. ${ }^{632}$ In patients with hypertensive heart disease, left ventricular diastolic dysfunction is observed from an early stage due to cardiac hypertrophy and myocardial fibrosis. Therefore, treatment for hypertension is expected to alleviate cardiac hypertrophy and myocardial fibrosis and improve diastolic function. In the EXCEED Study, the improvement in diastolic function was observed in proportion to the degree of decrease in blood pressure obtained by antihypertensive treatment in untreated hypertensive patients with left ventricular diastolic dysfunction. ${ }^{633}$ In addition, as tachycardia, particularly atrial fibrillation, often induces heart failure, its prevention and appropriate control of the heart rate are important. The possibility of diastolic dysfunction due to latent coronary artery diseases should also be considered. In a randomized study, ARBs did not improve the prognosis of heart failure with HFpEF. ${ }^{634-636}$ However, a large-scale, prospective study (Swedish Heart Failure Registry) indicated that the total mortality rate was lower in patients taking RA system inhibitors. ${ }^{637}$

\section{3) Cardiac hypertrophy}

Cardiac hypertrophy is caused by pressure load and often regresses through long-term antihypertensive treatment. Epidemiological studies have revealed that cardiac hypertrophy is one of the independent risk factors that determine the prognosis of hypertensive patients. The mortality rate and incidence of cardiac events or heart failure due to coronary artery disease are high in patients with cardiac hypertrophy ${ }^{638}$ The incidence of cardiac events and sudden death decreases in patients who show regression of cardiac hypertrophy by antihypertensive treatment compared with those who do not. ${ }^{639,640}$ The CASE-J subanalysis showed that, when a blood pressure of $<130$ / 75-79 mm Hg was achieved in hypertensive patients with left ventricular hypertrophy, the incidence of cardiovascular events could be reduced to the level in those without cardiac hypertrophy. ${ }^{641}$ Both systolic and diastolic blood pressures are significant factors for cardiac hypertrophy and must be controlled for the treatment of cardiac hypertrophy.

A meta-analysis directly comparing cardiac hypertrophy-regressing effects among various antihypertensive drugs indicated that RA system inhibitors and $\mathrm{Ca}$ channel blockers exhibited the most marked 
effects. ${ }^{405}$ There are also reports in Japan that a combination use of aldosterone antagonists with ACE inhibitors or ARBs is more effective for regression of cardiac hypertrophy. ${ }^{642,643}$ However, the most important factor in the regression of cardiac hypertrophy is a sufficient decrease in blood pressure, and hence all drugs used as the first choice today are expected to regress cardiac hypertrophy through sustained control of blood pressure. ${ }^{644,645}$

\section{KIDNEY DISEASE}

\section{POINT 6C}

1. In patients with CKD, risk factors for cardiovascular disease (CVD), such as hypertension and abnormal diurnal changes in blood pressure, are frequently observed, and the risk of cardiovascular accidents is high.

2. For the early detection of CKD, urinalysis and calculation of the estimated glomerular filtration rate (eGFR) should be performed in all hypertensive patients. In patients with diabetes mellitus (diabetes $(+)$ ), the urinary albumin level should be evaluated using the urinary albumin-to-urinary creatinine $(\mathrm{Cr})$ ratio ( $\mathrm{mg}$ per $\mathrm{g} \mathrm{Cr}$ ). In those without diabetes mellitus (diabetes $(-)$ ), the urinary protein-to-urinary creatinine ratio (gram per $\mathrm{g} \mathrm{Cr}$ ) should be measured when a qualitative test for urinary protein shows $( \pm)$ or higher, and patients with a value of 0.15 gram per gram $\mathrm{Cr}$ or above should be regarded as showing proteinuria (+).

3. Target of blood pressure control

In diabetes ( + ) patients, the target of blood pressure control should be $<130 / 80 \mathrm{~mm} \mathrm{Hg}$ regardless of the presence or absence of albuminuria (Recommendation grade: B, Evidence level: II). In diabetes (-) and proteinuria (-) patients, it should be $<140 / 90 \mathrm{~mm} \mathrm{Hg}$ (Recommendation grade: A, Evidence level: I). In those with proteinuria (+), it should be $<130 / 80 \mathrm{~mm} \mathrm{Hg}$ (Recommendation grade: C1, Evidence level: III). In elderly patients, an excessive decrease in blood pressure should be avoided.

4. Regarding lifestyle, salt restriction, maintenance of appropriate body weight, smoking cessation and restriction of protein intake in accordance with the renal function should be practiced. Salt intake should be $<6 \mathrm{~g}$ per day (Recommendation grade: A, Evidence level: I). However, it should not be reduced to $<3 \mathrm{~g}$ per day (Recommendation grade: $\mathrm{C} 2$, Evidence level: IVb).

5. When blood pressure exceeds the target level of blood pressure control, antihypertensive drug therapy should be promptly started, in addition to lifestyle modifications.

6. First-choice drugs

In diabetes (+) patients, RA system inhibitors should be selected regardless of the presence or absence of albuminuria (patients without albuminuria: Recommendation grade: C1, Evidence level: III; patients with albuminuria: Recommendation grade: A, Evidence level: I)). In diabetes (-) and proteinuria (-) patients, RA system inhibitors, Ca channel blockers or diuretics should be selected (Recommendation grade: B, Evidence level: II). In those with proteinuria (+), RA system inhibitors should be selected (Recommendation grade: $B$, Evidence level: II).

7. A decrease in urinary protein level is strongly associated with the prevention of end-stage renal disease. It is important to decrease the urinary protein level as much as possible.
8. In advanced CKD patients with a GFR of $<30 \mathrm{ml} \mathrm{min}^{-1}$ per $1.73 \mathrm{~m}^{2}$ or in elderly patients with CKD, RA system inhibitors should be started at a low dose. Changes in eGFR and serum $\mathrm{K}$ level must be monitored.

9. Combination therapy with several antihypertensive drugs is required in many cases. For the use of diuretics, thiazides should be selected if the GFR is $30 \mathrm{ml} \mathrm{min}^{-1}$ per $1.73 \mathrm{~m}^{2}$ or higher, and loop diuretics should be selected if the GFR is less than $30 \mathrm{ml} \mathrm{min}^{-1}$ per $1.73 \mathrm{~m}^{2}$.

10. Patients with CKD may develop acute renal dysfunction. They must be instructed to promptly consult a medical doctor if a possibility of dehydration is suspected in such cases as vomiting, diarrhea or fever.

1) Renal function and blood pressure

There is a close association between hypertension and kidneys. The kidney has an important role in the etiology of hypertension. On the other hand, hypertension causes renal dysfunction, leading to CKD. Once CKD develops, hypertension becomes severe, and a vicious circle is established. In CKD patients, abnormalities in the diurnal rhythm of blood pressure, such as the disappearance of a nocturnal decrease, are observed, becoming a risk factor for the onset of CVD. In addition, $\mathrm{CKD}$ is complicated by sleep apnea syndrome in a high proportion of patients, exacerbating hypertension. ${ }^{646,647}$ In addition to treatment for the primary disease of $\mathrm{CKD}$, strict management of blood pressure over $24 \mathrm{~h}$ is important.

Renal function declines with age after the 30s, but the rate of ageassociated decrease in the GFR estimated from the Japanese health screening data is very low (about $0.3 \mathrm{ml} \mathrm{min}^{-1}$ per year). ${ }^{648} \mathrm{On}$ the other hand, the GFR may decrease at a rate of $4-8 \mathrm{ml} \mathrm{min}^{-1}$ per year in hypertensive patients. ${ }^{649}$ The Japanese health screening data showed that aging, diabetes mellitus and hypertension were important risk factors for the appearance of proteinuria or for the occurrence of CKD with a GFR of $<60 \mathrm{ml}$ min per $1.73 \mathrm{~m}^{2} .22$

In Japan, major primary diseases in patients chronically undergoing dialysis include diabetic nephropathy, chronic glomerulonephritis and nephrosclerosis. The number of patients undergoing chronic dialysis has steadily increased, and diabetic nephropathy and nephrosclerosis are primary causes of this increase. In recent times, the number of patients for whom dialysis has been newly introduced due to chronic glomerulonephritis has decreased. ${ }^{650}$ Blood pressure and urinary protein levels are known to be strong factors contributing to the onset of end-stage renal disease (ESRD). In a prospective cohort study involving residents, the incidence of ESRD was the lowest in the optimal blood pressure group. It increased with the blood pressure level, and there was no J-shaped phenomenon. ${ }^{21,651}$

\section{2) Diagnosis of CKD and its significance}

Renal dysfunction and proteinuria are known to be risk factors for ESRD, ${ }^{652-655}$ but they have also recently been found to be strong risk factors for CVD. ${ }^{245,248,249,656-659}$ In order to prevent the occurrence of ESRD and CVD through the early detection/intervention of renal damages, the concept of CKD was proposed by the National Kidney Foundation in $2002 .{ }^{660} \mathrm{CKD}$ is defined as the presence of findings suggesting renal damages (abnormalities on urinalysis, abnormalities on imaging, or pathological findings) or renal dysfunction with a GFR of $<60 \mathrm{ml} \mathrm{min}^{-1}$ per $1.73 \mathrm{~m}^{2}$ for 3 months or more. ${ }^{439,661}$ According to epidemiological studies involving residents, even mild proteinuria and microalbuminuria were risk factors for CVD, ${ }^{662}$ and most patients with CKD died of CVD prior to the occurrence of ESRD. ${ }^{657,658}$ 
Therefore, the early detection of CKD is important. In all hypertensive patients, the eGFR should be calculated, and urinalysis should be performed. In Japan, urinary albumin measurement is covered by health insurance only in diabetics. In hypertensive patients without diabetes mellitus, the qualitative test of proteinuria is conducted. If the qualitative test shows $( \pm)$ or higher, proteinuria should be quantitatively evaluated using the urinary protein/urinary creatinine ratio. Patients with a value of $0.15 \mathrm{~g}$ per g Cr or more should be regarded as showing proteinuria $(+)$. In the Guidelines, both diabetics with microalbuminuria (30 mg per g Cr or more) and nondiabetics with mild proteinuria ( $0.15 \mathrm{~g}$ per $\mathrm{g} \mathrm{Cr}$ or more) are regarded as having proteinuria $(+)$. The quantification of urinary albumin/protein is also recommended from the perspective of treatment selection and evaluation of the treatment response.

In patients with CKD, a decrease in GFR and the presence of proteinuria are risk factors for the development of ESRD and CVD, respectively, through independent mechanisms. The risk increases as urinary protein excretion increases and GFR decreases. If the two factors coexist, the risk additively increases. ${ }^{248,249}$ In the Kidney Disease: Improving Global Outcomes (KDIGO) ${ }^{663}$ and the Guide for CKD Treatment published by the Japanese Society of Nephrology, ${ }^{439}$ a heat map to evaluate the risk based on a combination of a decrease in GFR and urinary protein (albumin) excretion was presented.

Primary diseases are involved in the pathophysiology of CKD. Among patients with the same degree of renal dysfunction, the incidence of CVD in patients with hypertensive nephrosclerosis or diabetic nephropathy is higher than in those with chronic glomerulonephritis. ${ }^{664}$ In the former, even mild proteinuria is a strong risk factor for CVD. In the presence of diabetes mellitus or hypertension, afferent arterioles exposed to high intravascular pressure are initially affected, and, subsequently, the downstream glomeruli are damaged, leading to the appearance of albuminuria. ${ }^{665}$ Therefore, there may be a close association between proteinuria and lesions of brain-perforating arteries, or coronary artery diseases, because both of these vasculatures are exposed to high intraluminal pressure as in the case of the afferent arterioles. ${ }^{666}$ Furthermore, the urinary protein level depends on the degree of damage of individual glomeruli and the number of affected glomeruli. If the urinary protein level is higher, a larger number of glomeruli may be markedly damaged. Severely damaged glomeruli would finally become extinct. Therefore, proteinuria is a strong predictive factor for the occurrence of renal insufficiency.

A recent epidemiological study showed that the prevalence of CKD was higher than expected. In Japan, the number of CKD patients is estimated to be $\sim 13000000$, accounting for about $13 \%$ of the adult population. ${ }^{667}$ In Japan, with aging of the population, the number of patients with lifestyle-related diseases such as obesity, hypertension and diabetes mellitus is increasing. Thus, the early detection, treatment and prevention of CKD are important.

\section{3) Diabetic nephropathy (CKD with diabetes mellitus)}

Diabetic nephropathy is the leading cause for initiation of dialysis in Japan, accounting for about $40 \%$ of new dialysis cases. ${ }^{650}$ Nephropathy is considered to be present in about $40 \%$ of diabetics in Japan. ${ }^{668}$ Some diabetics show a normal urinary albumin level but have a GFR of $<60 \mathrm{ml} \mathrm{min}^{-1}$ per $1.73 \mathrm{~m}^{2}$. 669 There are many patients with type 2 diabetes and hypertension. When hypertensive nephrosclerosis is a primary lesion, the urinary protein level is relatively low. Albuminuria has been found to have a particularly important role in the onset of renal insufficiency or CVD in CKD patients with diabetes mellitus. Urinary albumin should be quantitatively evaluated regularly using its ratio to urinary creatinine and used as an index of treatment.
Recently, it has also been shown in Japan that the progression of nephropathy can be prevented and its remission or regression achieved by intensive treatment, and that the remission or regression of diabetic nephropathy is closely related to the prevention of CVD as well as renal insufficiency. ${ }^{670}$ INNOVATION ${ }^{463}$ indicated that, in addition to a sufficient reduction in blood pressure, the administration of RA system inhibitors was necessary for inducing remission and regression. ARBs at a high dose are particularly effective for advanced microalbuminuria with a urinary albumin level of $100-299 \mathrm{mg}$ per $\mathrm{g}$ Cr. ${ }^{463,671}$ The doses of RA system inhibitors should be increased using urinary albumin excretion as an index, while monitoring changes in the renal function.

\section{4) Lifestyle modifications}

An inappropriate lifestyle is the most important reason for the present increase in CKD patients. Obesity and an excessive salt intake accelerate kidney damage by mechanisms dependent on and independent of blood pressure. Lifestyle modifications are the most basic and important factors in the treatment of $\mathrm{CKD}$, in which restricting salt intake, maintaining an appropriate body weight, cessation of smoking and restricting protein intake are essential.

Restriction of salt intake is important for controlling blood pressure and for preventing the progression of renal dysfunction. As salt sensitivity is often enhanced in hypertensive patients with CKD, restriction of salt intake would be effective for reducing blood pressure. Salt restriction enhances the hypotensive and antiproteinuric effects of ACE inhibitors and ARBs. ${ }^{672}$ A subanalysis of a study involving patients with diabetic nephropathy showed that the incidences of ESRD and CVD decreased in the low salt intake group, whereas they increased in the extremely low salt intake group. ${ }^{673}$ The REIN Study indicated that the renoprotective effects of salt restriction were closely associated with a decrease in the urinary protein level. ${ }^{674}$ On the basis of these results, salt intake should be restricted to $<6 \mathrm{~g}$ per day (Grade A). However, salt restriction to $<3$ g per day should be avoided (Grade C2). It is important to promote salt restriction step by step while monitoring blood pressure and urinary protein excretion. In particular, caution is needed in elderly patients.

Abdominal obesity is involved in the development of ESRD or proteinuria. ${ }^{372,675,676}$ As a risk factor for CKD, body weight changes are important rather than body weight itself. ${ }^{677}$ Weight loss achieved by interventions reduces albuminuria, ${ }^{678,679}$ but no interventional study has yet investigated its long-term effects on renal function. Several studies have reported that abdominal obesity is significantly associated with the total mortality rate and incidence of CVD in CKD patients with an eGFR of $30 \mathrm{ml} \mathrm{min}^{-1}$ per $1.73 \mathrm{~m}^{2}$ or higher. ${ }^{680,681} \mathrm{On}$ the other hand, the relationship between obesity and prognosis remains to be clarified in CKD patients with an eGFR of $<30 \mathrm{ml} \mathrm{min}^{-1}$ per $1.73 \mathrm{~m}^{2}$. Thus, in CKD patients with an eGFR of $30 \mathrm{ml} \mathrm{min}{ }^{-1}$ per $1.73 \mathrm{~m}^{2}$ or higher, decreases in body weight/visceral fat induced by a decrease in energy intake may prevent the progression of $\mathrm{CKD}$, improving the prognosis; thus, weight reduction is recommended in obese patients (Grade C1). However, sufficient guidance should be conducted so that excessive dietary restrictions may not result in a deficiency of essential nutrients, such as vitamin.

Smoking has been reported to exert adverse effects on proteinuria and renal dysfunction in both diabetic and nondiabetic nephropathy patients. ${ }^{62,683}$ It has been established that smoking is a risk factor for CVD. Considering that the risk of cardiovascular death is high in CKD patients, smoking cessation is crucial.

A study indicated that restriction of protein intake decreased the incidence of ESRD and relative risk of death. ${ }^{684}$ However, restriction 
of protein intake does not influence the rate of reduction in GFR, although it prolongs the interval until renal replacement therapy is required. ${ }^{685-687}$ Strict restriction of protein intake induces various risks. In particular, in elderly patients with a low urinary protein level, the rate of reduction in renal function is essentially slow, and restriction of protein intake is not clinically relevant. Therefore, uniform guidance for restriction of protein intake is inappropriate, and guidance should be performed by comprehensively evaluating individual patients' conditions, risks and adherence. As standard treatment, protein intake is restricted to $0.6-0.8 \mathrm{~g}$ per $\mathrm{kg}$ standard body weight per day, but protein restriction may be started at $0.8-1.0 \mathrm{~g}$ per day in patients with mild renal dysfunction. ${ }^{439,661}$

There is insufficient evidence on exercise therapy, but guidance should be given in accordance with the renal function. ${ }^{68}$ Even in CKD patients with a GFR of $<60 \mathrm{ml} \mathrm{min}^{-1}$ per $1.73 \mathrm{~m}^{2}$ (including those undergoing dialysis), exercise therapy reduces blood pressure and central blood pressure and enhances cardiopulmonary functions, improving the quality of life. However, it does not influence the occurrence of CVD or rate of reduction in renal function. ${ }^{689}$ On the other hand, exercise may increase the incidence of adverse events in CKD patients with a GFR of $<60 \mathrm{ml} \mathrm{min} \mathrm{m}^{-1}$ per $1.73 \mathrm{~m}^{2}$. Therefore, in CKD patients, exercise therapy should be performed after sufficiently understanding the individual's clinical background. However, excessive exercise restriction should be avoided because it induces obesity and affects blood glucose/blood pressure control.

\section{5) Treatment with antihypertensive drugs}

The objective of antihypertensive treatment in CKD patients is to inhibit or prevent the progression of renal dysfunction and to prevent the occurrence or recurrence of CVD by reducing blood pressure. For this treatment, it is important to establish the most appropriate target level of blood pressure control for individual patients and perform adequate, individual-matched antihypertensive treatment, considering the patient's background (age, complications and daily environment). From the perspective of simultaneous brain/heart/kidney protection, the following points should be targeted: (1) achieving the target of blood pressure control and (2) reducing or, if possible, normalizing urinary protein (albumin) excretion. To achieve these objectives, combination therapy with RA system inhibitors is necessary in many cases. On the other hand, many CKD patients are elderly and often have complications, such as diabetes mellitus and sclerotic lesions of the coronary artery/cerebral blood vessels/renal artery; in these patients, RA system inhibitors or diuretics may induce an excessive decrease in blood pressure or acute renal dysfunction. To avoid such adverse events and achieve effective antihypertensive treatment, it is essential for individual patients to practice home blood pressure measurement and understand the treatment being received as well as the symptoms associated with its adverse effects. In particular, attention must be paid to the rate of decrease in blood pressure in elderly patients, and an excessive decrease in blood pressure should be avoided.

There is much evidence that antihypertensive treatment prevents the progression of CKD and reduces the risk of CVD and death. To prevent the progression of CKD and occurrence of CVD, strict, 24-h blood pressure control is essential. In the JSH2009 Guidelines, the target of blood pressure control in CKD patients was established as $<130 / 80 \mathrm{~mm} \mathrm{Hg}$, and as $<125 / 75 \mathrm{~mm} \mathrm{Hg}$ if the urinary protein level is $1 \mathrm{~g}$ per day or higher. However, recently, the opinion that 'there is no positive reason for lowering blood pressure to $<130 / 80 \mathrm{~mm} \mathrm{Hg}$ ' was reported by the ESH through a reassessment of clinical studies. ${ }^{690}$ In addition, a J-shaped phenomenon in which the incidence of myocardial infarction and mortality rate increase with an excessive decrease in blood pressure may occur in patients with coronary artery disease (ischemic heart disease). ${ }^{284,285}$ Based on this background, the ESH/ ESC 2013 Guidelines recommend the target of blood pressure control in CKD patients to be $<140 / 90 \mathrm{~mm} \mathrm{Hg}$, and only when overt proteinuria is present may a systolic blood pressure of $130 \mathrm{~mm} \mathrm{Hg}$ be targeted while monitoring changes in the GFR. ${ }^{120}$

The targets of blood pressure control and first-choice drugs in the Guidelines are shown in Table 6-3. This target of blood pressure control reflects that the incidence of stroke is high in Japanese patients with CKD, that the risk of CVD is high in diabetics and that the presence of proteinuria is a strong risk factor for ESRD and CVD. Antihypertensive drug therapy should be promptly started with lifestyle modifications if blood pressure exceeds the target of blood pressure control. Even when the blood pressure level is high-normal, treatment with RA system inhibitors should be started to reduce the urinary protein (albumin) level while monitoring blood pressure and urinary protein.

The results of the ADVANCE and observational studies in Japan have suggested that reducing blood pressure to $<130 / 80 \mathrm{~mm} \mathrm{Hg}$ in diabetic patients prevents the progression of CKD. ${ }^{691,692}$ On the other hand, in the ACCORD Study, albuminuria was markedly reduced in the strict blood pressure control group, but the eGFR was more favorably maintained in the standard blood pressure control group. ${ }^{285}$ With respect to the level of blood pressure control and CVD, the IDNT Study indicated that the total mortality rate was the lowest in patients with a systolic blood pressure of $121-130 \mathrm{~mm} \mathrm{Hg}$ and that it increased in those with a systolic blood pressure of $120 \mathrm{~mm} \mathrm{Hg}$ or below. ${ }^{693}$ On the other hand, a meta-analysis of 13 interventional studies including the ACCORD Study showed that there were no preventive effects of strict blood pressure control on composite outcomes of CVD events, whereas it prevented stroke. ${ }^{258}$ According to the CASE-J ${ }^{641}$ and Challenge-DM ${ }^{694}$ Studies, the occurrence of CVD was lowest in the group in which a clinic blood pressure of $<130 /$ $80 \mathrm{~mm} \mathrm{Hg}$ was achieved.

Table 6-3 Target of blood pressure control and first-choice drugs in patients with chronic kidney disease

\begin{tabular}{|c|c|c|c|c|}
\hline & & & Target of blood pressure control & First-choice drugs \\
\hline Diabetes mellitus (+) & & & $<130 / 80 \mathrm{~mm} \mathrm{Hg}$ & RA system inhibitors \\
\hline \multirow[t]{2}{*}{ Diabetes mellitus $(-)$} & Urinary protein & Absent & $<140 / 90 \mathrm{~mm} \mathrm{Hg}$ & $\begin{array}{l}\text { RA system inhibitors, Ca channel } \\
\text { blockers, diuretics }\end{array}$ \\
\hline & Urinary protein & Present & $<130 / 80 \mathrm{~mm} \mathrm{Hg}$ & RA system inhibitors \\
\hline
\end{tabular}

Urinary protein: patients with a urinary protein level of $0.15 \mathrm{mg}$ per gram of $\mathrm{Cr}$ or higher are regarded as having urinary protein.

In patients with a glomerular filtration rate of $<30 \mathrm{ml} \mathrm{min}^{-1}$ per $1.73 \mathrm{~m}^{2}$ or elderly patients, the administration of renin-angiotensin system inhibitors should be started at a low dose.

Diuretics: Thiazides should be selected if the glomerular filtration rate is $30 \mathrm{ml} \mathrm{min}^{-1}$ per $1.73 \mathrm{~m}^{2}$ or higher, and long-acting loop diuretics should be selected if the glomerular filtration rate is

$<30 \mathrm{ml} \mathrm{min}^{-1}$ per $1.73 \mathrm{~m}^{2}$.

Diabetics or chronic kidney disease patients with proteinuria who show a blood pressure of $130 / 80 \mathrm{~mm} \mathrm{Hg}$ or above are clinically regarded as having hypertension. 
The target of blood pressure control in CKD patients without diabetes mellitus should be $<140 / 90 \mathrm{~mm} \mathrm{Hg}$ in the absence of proteinuria and $<130 / 80 \mathrm{~mm} \mathrm{Hg}$ in its presence. Only a limited number of studies, such as the AASK, ${ }^{695}$ MDRD $^{696}$ and REIN-2, ${ }^{697}$ have examined the relationship between the level of blood pressure control and renal events in CKD patients without diabetes mellitus. There were no differences in composite renal end points between the standard and strict blood pressure control groups. In the KEEP Observational Study involving CKD patients with an eGFR of $<30 \mathrm{ml} \mathrm{min}^{-1}$ per $1.73 \mathrm{~m}^{2}$, the incidence of ESRD was the lowest in patients with a systolic blood pressure of $130-140 \mathrm{~mm} \mathrm{Hg}{ }^{698}$ On the basis of these results, blood pressure should be maintained at $<140$ / $90 \mathrm{~mm} \mathrm{Hg}$ from the perspective of the prevention of CKD progression (Grade A). On the other hand, subanalyses of the AASK and MDRD studies and follow-up studies after the completion of these studies showed that strict blood pressure control retarded deterioration of renal function only in patients with proteinuria. ${ }^{699-702}$ Furthermore, a meta-analysis of 11 randomized studies also indicated that strict blood pressure control was useful for reducing the rate of decline in renal function in patients with proteinuria. ${ }^{703}$ In an observational study in Japan, which examined the relationship between the occurrence of CVD and blood pressure level in CKD patients, the incidences of CVD in the high-normal blood pressure and normotensive groups were lower than those in the hypertensive group. ${ }^{32}$ In the ARIC and CHS Studies involving CKD patients with an eGFR of $15-60 \mathrm{ml} \mathrm{min}^{-1}$ per $1.73 \mathrm{~m}^{2}$, the risk of stroke was the lowest in patients with a systolic blood pressure of $120-129 \mathrm{~mm} \mathrm{Hg}$, and it increased in those with a systolic blood pressure of $<120 \mathrm{~mm} \mathrm{Hg}$ and $\geqslant 130 \mathrm{~mm} \mathrm{Hg} .{ }^{704}$ On the other hand, in the PROGRESS Study, a subanalysis of CKD patients (defined on the basis of eGFR alone) showed that the recurrence of stroke was more markedly inhibited in patients in whom a lower target level of blood pressure control was achieved regardless of baseline blood pressure. ${ }^{705}$ Furthermore, a subanalysis of CASE-J indicated that the incidence of CVD was the lowest in patients in whom blood pressure was controlled at $<130 / 80 \mathrm{~mm} \mathrm{Hg}$. These results are based on observational studies or subanalyses, and the evidence level is not high. However, considering that the incidence of stroke is high in the Japanese population, and that proteinuria is a risk factor for stroke independent of renal function, ${ }^{706}$ the target of blood pressure control in proteinuria (+) patients is established as $<130 /$ $80 \mathrm{~mm} \mathrm{Hg}$ in the Guidelines from the perspective of kidney protection and CVD prevention (Recommendation grade: C1).

CKD patients show abnormal diurnal changes in blood pressure. Nighttime hypertension is a risk factor for the progression of CKD and occurrence of CVD. These patients must be instructed to measure home blood pressure in the morning and at night, and, if necessary, ABPM should be performed. Antihypertensive therapy should be conducted prudently by adjusting the time of dosing. Moreover, if diurnal changes are present, screening of sleep apnea syndrome is necessary. If it is present, treatment should be performed.

Proteinuria not only indicates glomerular or vascular damage but is also considered to exacerbate the renal function. In patients with proteinuria, RA system inhibitors may particularly exhibit organprotecting effects ${ }^{695,707,709}$ and are selected as a first-choice drug. To sufficiently decrease urinary protein excretion, it is necessary to adjust the doses of ACE inhibitors or ARBs in addition to strict blood pressure control. A subanalysis of CASE-J also showed that ARBs more markedly prevented the onset of CVD and renal events compared with Ca channel blockers in CKD patients with an eGFR of 15$30 \mathrm{ml} \mathrm{min}^{-1}$ per $1.73 \mathrm{~m}^{2}$. With regard to renoprotective effects, ARBs were more advantageous only in patients with proteinuria. ${ }^{709}$ On the other hand, the efficacy of RA system inhibitors has not been established in CKD patients without proteinuria, and RA system inhibitors, Ca channel blockers or diuretics may become a first-choice drug. For the use of diuretics, thiazide diuretics should be selected in patients with a GFR of $30 \mathrm{ml} \mathrm{min}^{-1}$ per $1.73 \mathrm{~m}^{2}$ or higher, and loop diuretics should be selected in those with a GFR of $<30 \mathrm{ml} \mathrm{min}^{-1}$ per $1.73 \mathrm{~m}^{2}$.

RA system inhibitors can be administered to patients with CKD regardless of the stage, but rapid deterioration of the renal function or hyperkalemia is observed in some elderly patients or CKD patients with a GFR of $<30 \mathrm{ml} \mathrm{min}^{-1}$ per $1.73 \mathrm{~m}^{2}$. Therefore, administration should be started at a low dose, and the renal function and serum $\mathrm{K}$ level must be closely monitored. Usually, RA system inhibitors slowly exhibit hypotensive effects, and there is no rapid decrease in blood pressure after administration. If there is a rapid decrease in blood pressure, this may be associated with dehydration, excessive salt restriction, high-dose therapy with diuretics or renal artery stenosis. To detect a rapid decrease in blood pressure, home blood pressure measurement is effective. If an excessive decrease in blood pressure (systolic blood pressure: $30 \mathrm{~mm} \mathrm{Hg}$ or greater) is noted immediately after administration, its etiology must be evaluated, and the patient should be referred to a specialist if necessary.

RA system inhibitors ameliorate glomerular hypertension/hyperfiltration by reducing the systolic blood pressure and dilating the efferent arterioles, and therefore the GFR may decrease occasionally. However, this decrease is a reflection of functional change rather than the progression of renal tissue damage, because the GFR returns to its previous level on discontinuing the drug. ${ }^{710}$ As there is also a report that in those whose renal function, renal function declined temporarily shortly after the beginning of the administration was well maintained over a long period thereafter, careful observation is recommended when an initial increase in the serum creatinine level is mild (rate of decrease in the GFR: $<30 \%$ ). As a decrease in renal function usually becomes apparent within a few days after commencing administration, the serum creatinine level should be measured before and 2 weeks ( 1 week if possible) after the first administration. If exacerbation of renal function is noted, its causes, such as bilateral renal artery stenosis, should be sought. An increase in the serum $\mathrm{K}$ level may also be observed, and its treatment comprises the concomitant use of a diuretic or administration of sodium bicarbonate. The administration of nonsteroidal anti-inflammatory drugs (NSAIDs) should be avoided, because they exacerbate renal function and increase the serum K level. Added to this, as ACE inhibitors are excreted via the kidney, with some exceptions, their dose adjustment is necessary in patients with reduced renal function. However, dose adjustment is mostly unnecessary for ARBs, which are excreted via the bile.

In many patients with $\mathrm{CKD}$, multiple-drug combination therapy is necessary to achieve the target blood pressure. ${ }^{649}$ In the ACCOMPLISH Study, the incidence of renal events in patients undergoing combination therapy with an ACE inhibitor and a long-acting $\mathrm{Ca}$ channel blocker was lower than in those undergoing combination therapy with an ACE inhibitor and a thiazide diuretic. ${ }^{438}$ A CKD subanalysis of the OSCAR Study involving elderly patients showed that, compared with the ARB alone at an increased dose, a combination therapy of an ARB and a Ca channel blocker resulted in better blood pressure control and a low incidence of CVD. ${ }^{711}$ Ca channel blockers have various characteristics. Clinical studies have indicated that the urinary protein-decreasing effects of some Ca channel blockers are similar to those of ACE inhibitors. ${ }^{712-714}$ Other studies reported that, when added to RA system inhibitors, the antiproteinuric effect differed among Ca channel blockers. ${ }^{430,452,715}$ On the 
other hand, diuretics are required in advanced CKD patients in whom body fluid management is difficult. The principal strategy of body fluid management is salt restriction. Diuretics should be administered at doses as low as possible. In the case of intensive treatment with diuretics, caution should be taken against electrolyte abnormalities, such as hypokalemia, or dehydration. Thus, Ca channel blockers are generally recommended as a drug to be combined with an RA system inhibitor, but, if an excessive volume of body fluid such as edema is observed, low-dose diuretics should be predominantly used. If blood pressure does not reach its target level despite a combination of two drugs, combination therapy with three drugs, an RA system inhibitor, a Ca channel blocker and a diuretic, should be performed, or the doses of an RA system inhibitor and a Ca channel blocker should be increased. If the target of blood pressure control is not achieved despite these approaches, the patient should be regarded as having resistant hypertension (see the corresponding chapter) and referred to a specialist if necessary.

It has been reported that combination therapy with an ARB and an ACE inhibitor reduces the urinary protein level. However, this combination may increase the risk of acute renal dysfunction; thus, caution is needed. Although aldosterone antagonists decrease the urinary protein level, ${ }^{716,717}$ they increase the risk of hyperkalemia when combined with RA system inhibitors. Therefore, this combination therapy should be carefully performed at a very low dose while monitoring the serum $\mathrm{K}$ level. Eplerenone is contraindicated for patients with diabetic nephropathy and for nondiabetic CKD patients with a Ccr of $<50 \mathrm{ml} \mathrm{min}^{-1}$ per $1.73 \mathrm{~m}^{2}$.

Direct renin inhibitor (DRI) increases the renal blood flow. The additional administration of this drug to patients with overt diabetic nephropathy receiving high-dose ARB therapy reduces urinary albumin excretion without decreasing the eGFR. ${ }^{489}$ However, evidence regarding DRI is insufficient in hypertensive patients with CKD. Recently, the results of the ALTITUDE Study, in which a DRI, aliskiren, was additionally administered to high-risk nephropathy patients with type 2 diabetes taking RA system inhibitors at a high dose, were published. ${ }^{490}$ There were no differences in the incidence of CVD or renal end points between the aliskiren and placebo groups. In the former, adverse events were more frequent. On the basis of the results of the study, combination therapy with a DRI and other RA system inhibitors is generally contraindicated for diabetic nephropathy and nondiabetic CKD patients with an eGFR of $<60 \mathrm{ml} \mathrm{min}^{-1}$ per $1.73 \mathrm{~m}^{2}$. This combination may only be selected under a specific condition (when blood pressure control is not sufficient).

\section{6) Patients undergoing dialysis}

In patients undergoing hemodialysis, there is little evidence regarding the appropriate target of blood pressure control or selection of antihypertensive drugs. Moreover, blood pressure measurement has not been standardized. In patients undergoing hemodialysis, the relationship between blood pressure level and prognosis markedly differs from that in the general population, showing a U-shaped pattern. The mortality rate was the lowest in patients with a systolic blood pressure of $120-160 \mathrm{~mm} \mathrm{Hg}$ before dialysis. ${ }^{718-721}$ Malnutrition is not uncommon in patients treated with maintenance hemodialysis, and the incidence of complications such as heart failure is high. The association between blood pressure alone and prognosis is difficult to clarify. ${ }^{722}$ In addition, a study reported that there were marked changes in blood pressure related to hemodialysis and that a decrease in blood pressure during dialysis and the presence of orthostatic hypotension immediately after dialysis were independent risk factors for total mortality. ${ }^{723}$
The primary mechanism for hypertension in patients undergoing dialysis is an excessive volume of body fluid. Therefore, it is most important to optimize the dry weight (body weight at which there are minimal signs or symptoms of hypovolemia or hypervolemia), which is measured by a dialysis specialist, and reduce weight gain between dialysis points. ${ }^{724}$ In patients undergoing dialysis, blood pressure changes periodically, as the body fluid volume decreases on every session of dialysis and the body weight increases before the subsequent dialysis. There are various measurement points of blood pressure: before dialysis, during dialysis, after the completion of dialysis, on standing after its completion, and average weekly blood pressure. ${ }^{725}$ Blood pressure assessment, involving nondialysis days, not only in the dialysis room but also at home is important.

Thus, blood pressure is exposed to a specific environment in patients undergoing dialysis, and blood pressure management is basically performed by dialysis specialists. The 'Guidelines for the Evaluation and Treatment of Cardiovascular Complications in Patients on Hemodialysis' were prepared by the Japanese Society for Dialysis Therapy, describing blood pressure measurement in patients undergoing dialysis, the target of blood pressure control, timing of the administration of antihypertensive drugs and selection of antihypertensive drugs. ${ }^{726}$ Therefore, currently, dialysis specialists manage blood pressure in accordance with individual dialysis patients' conditions according to the guidelines, and consult specialists if necessary.

\section{VASCULAR DISEASES}

\section{POINT 6D}

Vascular diseases

1. Acute aortic dissection requires immediate blood pressure reduction and pain control. The systolic blood pressure should be controlled at $100-120 \mathrm{~mm} \mathrm{Hg}$ (Recommendation grade: $\mathbf{C} 1$, Evidence level: III).

2. In patients with chronic aortic dissection, the target of systolic blood pressure control should be 130 to $<135 \mathrm{~mm} \mathrm{Hg}$ (Recommendation grade: C1, Evidence level: VI).

3. In patients with atherosclerotic peripheral arterial disease, a supervised exercise training program is recommended. Added to this, intensive risk factor management including strict control of blood pressure is expected to reduce the concurrence of cardiovascular events.

\section{1) Aortic aneurysm}

(1) Aortic dissection. Acute aortic dissection is a hypertensive emergency that requires immediate blood pressure reduction, control of the heart rate, pain control and complete rest. The site and morphology of dissection and the presence or absence of peripheral circulatory disorders due to stenosis/obstruction of arteries branching from the aorta should be evaluated continuously and carefully. As a rule, surgery should be promptly performed to treat Stanford type A dissection, and drug therapy should be administered to treat Stanford type $\mathrm{B}$ dissection. ${ }^{727}$

For blood pressure control, systolic blood pressure should be maintained at $100-120 \mathrm{~mm} \mathrm{Hg}$ by the continuous infusion of a $\mathrm{Ca}$ channel blocker (nicardipine, diltiazem), nitroglycerin or nitroprusside in combination with a $\beta$-blocker, but there is no evidence regarding the target systolic blood pressure or effects of combination therapy with a $\beta$-blocker. ${ }^{727}$ When combining diltiazem with a $\beta$ blocker, bradycardia must be considered. 
There is no established evidence regarding the target systolic blood pressure or selection of antihypertensive drugs in patients with chronic aortic dissection. However, several studies have indicated that $\beta$-blockers decrease the number of dissection-associated accidents such as admission, ${ }^{728,729}$ and others have reported that ACE inhibitors prevent the rupture of abdominal aortic aneurysms. ${ }^{730}$ To prevent re-dissection or rupture, the target systolic blood pressure should be 130 to $<135 \mathrm{~mm} \mathrm{Hg}$. ${ }^{731}$

(2) Aortic aneurysm. As aortic aneurysm is asymptomatic in most patients, it is often detected incidentally on health screening or on examination for other diseases. However, once it ruptures, the mortality rate is very high, and even if patients come to the hospital in a stage of threatened rupture the survival rate is low because of unstable hemodynamics. ${ }^{732}$

Strict antihypertensive treatment for thoracic aortic aneurysm is important, and systolic blood pressure should be maintained at 105$120 \mathrm{~mm} \mathrm{Hg}$, although no evidence regarding the target of blood pressure control has been established. As for the selection of antihypertensive drugs, there is a report of a randomized controlled trial in which the administration of $\beta$-blockers was effective for the prevention of aneurysm enlargement in patients with Marfan's syndrome. ${ }^{733}$ Recently, a cohort study reported that ARBs were useful for inhibiting an increase in the aneurysmal diameter in 18 children with Marfan's syndrome. ${ }^{734}$

However, there is no established evidence regarding the effects of strict antihypertensive therapy or $\beta$-blockers on abdominal aortic aneurysm. In patients admitted with a diagnosis of abdominal aortic aneurysm, the frequency of ruptured aneurysm was reported to be significantly lower in those who received ACE inhibitors before admission in a recent large-scale case-controlled study. ${ }^{730}$ Undoubtedly, atherosclerosis is closely associated with the etiology of abdominal aortic aneurysm. Whereas the effectiveness of internal treatment for the prevention of enlargement or rupture of aneurysm has not been confirmed by large-scale randomized controlled studies, the importance of smoking cessation has been reported. ${ }^{735}$

Ultrasonography is useful for the diagnosis of abdominal aortic aneurysm. In addition, it is also useful for evaluating the aneurysmal diameter. If there is a slight increase in the aneurysmal diameter, surgery should be considered at an appropriate time. ${ }^{736}$

\section{2) Atherosclerotic peripheral arterial disease}

Peripheral circulatory disorders due to atherosclerotic vascular lesions are classified according to their severity into Fontaine grade I (no symptom, numbness, coldness), grade II (intermittent claudication), grade III (pain at rest) and grade IV (gangrene/ischemic ulcer). The objectives of treatment are the alleviation of symptoms of ischemia and prevention of cardiovascular events, which often complicate peripheral circulatory disorders. Systematic execution of an exercise program under supervision has been reported to be effective for alleviating ischemic symptoms in the lower limbs. ${ }^{737}$ Strict blood pressure control is more important for preventing cardiovascular events rather than for improving ischemic symptoms in the lower limbs. ${ }^{738}$ Therefore, appropriate antihypertensive drugs should be selected according to the complications or patient's conditions that require careful use of drugs (see Chapter 5, Treatment with antihypertensive drugs). The administration of ACE inhibitors to patients with symptomatic atherosclerotic peripheral arterial disease has been reported to have suppressed cardiovascular events by about $25 \%$ in a large-scale randomized controlled study. ${ }^{556} \beta$-Blockers have been considered to exacerbate ischemic symptoms in the lower limbs; however, their safe use has been reported in a randomized study involving patients with intermittent claudication. ${ }^{739,740}$ They can be used in patients with heart failure or coronary artery disease, which often complicate atherosclerotic peripheral arterial disease. Physicians should refer patients with severe atherosclerotic peripheral arterial disease to a specialist, because percutaneous transluminal angioplasty or surgical circulatory reconstruction is required in some cases.

\section{Citation Information}

We recommend that any citations to information in the Guidelines are presented in the following format:

The Japanese Society of Hypertension Guidelines for the Management of Hypertension (JSH2014). Hypertens Res 2014; 37: 253-392.

Please refer to the title page for the full list of authors. 